\title{
Robust Excitation Force Estimation and Prediction for Wave Energy Converter M4 Based on Adaptive Sliding-Mode Observer
}

\author{
Yao Zhang, Tianyi Zeng, and Guang Li, Member, IEEE
}

\begin{abstract}
The wave excitation force estimation and prediction plays an important role in improving the performance of causal and non-causal controllers for wave energy converters (WECs). This paper proposes a robust adaptive sliding-mode observer (ASMO) to estimate the wave excitation force subject to unknown disturbances and parametric uncertainties for a multi-motion multi-float WEC, called M4. Both the convergence time and the estimation error can be explicitly bounded within expected limits by tuning the ASMO parameters, which are essentially beneficial for causal controllers to maintain the control performance. A fixed-time convergent sliding variable is designed to drive the estimation error into a small region within a fixed time. Due to the adaptive law, the overall system is proven to be finite-time stable, which allows explicit formulations of the convergence time and the estimation error. Moreover, based on the wave force estimation by the ASMO, an improved AutoRegressive (AR) model whose coefficients are updated by online training is developed to predict the wave excitation force. The prediction errors can also be explicitly estimated to achieve guaranteed control performance for the non-causal controller requiring future excitation force. From the comparison based on a realistic sea wave gathered from Cornwall, UK, it can be found that compared with the conventional Kalman Filter, the ASMO achieves a smaller steady-state estimation error and has satisfactory robustness performance against $30 \%$ model mismatch.
\end{abstract}

Index Terms-Wave Excitation Force, Wave Energy Converters, M4, Robustness, Sliding Mode Observer

\section{INTRODUCTION}

Sea waves contain enormous untapped renewable energy. Compared with other renewable energy sources such as wind energy and solar energy, sea waves offer high energy density and continuous power supply [1], [2].

To harness wave power, many types of wave energy converters (WECs) have been developed, including oscillating water column, overtopping, point absorber and attenuator. Control plays a key role in improving their energy conversion efficiency to reduce the unit cost of electricity. The information of the wave excitation force is normally required for implementation of some recently developed advanced WEC control strategies [3], and the accuracy of the wave excitation force significantly affects the control performance. Furthermore,

Y. Zhang is a postdoctoral researcher with Queen Mary University of London, UK, E1 4NS. e-mail: yao.zhang@qmul.ac.uk.

T. Zeng is with Beijing Institute of Technology, China, 100081. e-mail: tyzeng0525@outlook.com.

G. Li is with Queen Mary University of London, UK, E1 4NS. e-mail: g.li@qmul.ac.uk.

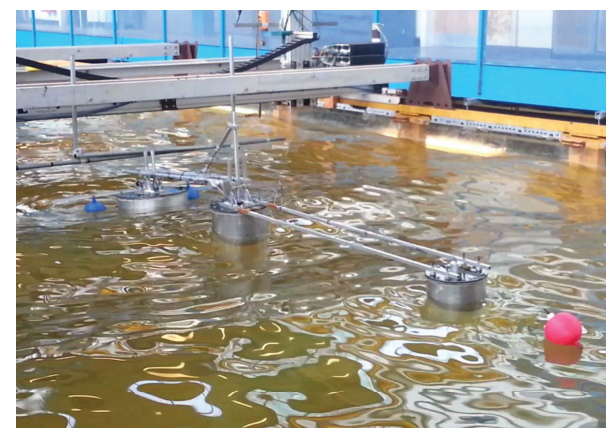

Fig. 1. Tank experiment of M4 in Manchester [9]

the wave excitation force prediction is also needed by noncausal WEC controllers [4] which incorporate the future wave information into the current control decision and the accuracy of wave force prediction relies on the estimation at the current time [5]. A direct measurement of wave excitation force by pressure sensors placed on the WEC wet-surface is reported in [6], while it is neither economically viable nor accurate in many cases.

The Kalman Filter (KF) was employed for wave excitation force estimation problem in recent years [7], [8] and was proven to be effective to achieve real-time estimations. While there is model mismatch between the state-space model and the original hydrodynamic model, which is caused by wave force approximations, unmodelled wave forces and environmental uncertainties, etc. Since the excitation force estimators are designed based on a state-space model subject to parametric uncertainties and unknown disturbances, which are significant in high sea states and degrade the control performance to some extend, this paper proposes a novel estimator based on the sliding mode technique that can cope with the model uncertainty and is helpful to improve the control performance. To be specific, with a better estimation and prediction of the excitation force, the controller can be designed based on a more accurate model, which enhances the control performance of energy maximization. Furthermore, the estimation error is also explicitly bounded by a prescribed limit.

The sliding-mode variable structure method is a well-known approach to cope with disturbances and modelling uncertainties [10]-[12] and has been widely applied in many fields. The sliding mode observer (SMO) ensures the robustness of the system when the modelling uncertainty exists and 
a short convergence time can be achieved when combined with a finite-time stabilization technique [13]. Since the SMO has been successfully applied in aerospace engineering [14], marine engineering [15] and permanent magnet synchronous motor system [16], etc., it has the potential to be used for the wave excitation force estimation subject to sea-state environmental uncerttainties. Due to these motivations, this paper proposes an adaptive sliding mode observer (ASMO) to improve the estimation performance of wave excitation forces. The sliding mode method and the adaptive method are employed together to enhance the estimation accuracy and the fixed-time stabilization method is adopted to ensure the explicit formulation of the boundaries of the convergence time and the estimation error.

The main contributions and novelties of the proposed ASMO are as follows:

- The convergence time of wave excitation force estimation and the boundary of estimation error are explicitly limited in prescribed boundaries, which are essentially beneficial for a WEC controller to achieve its guaranteed performance;

- The robustness of the ASMO can be guaranteed by sliding mode method and adaptive method, which enhances the control performance subject to modelling uncertainties;

- Based on the latest historical wave force estimation by the ASMO, an improved AR model which can continuously take into account the change of sea states is adopted to predict the excitation force. The prediction errors at each step can also be explicitly bounded, which can improve the control performance of non-causal WEC controllers;

- The coefficients of the improved AR model are trained online by solving a lease square (LS) problem, which is computationally cheap.

To demonstrate the efficacy of the proposed wave excitation force estimation method, we use an attenuator type WEC, called M4, as a case study [17]. M4 is a multi-float multimotion WEC, which exhibits more complicated dynamics than single float and single motion point absorbers. This is essentially a raft-type attenuator device which absorbs energy from the relative pitch motion of floats connected by beams at a hinge. There are three rows of floats with a single bow float attached to a mooring buoy; the floats increase in size downwave and the device heads naturally into the wave direction [18]. The point absorbers have been extensively studied in WEC control community and are also mainly used as benchmark problem for wave excitation force estimation. The observer design of multi-float multi-motion WECs has not been investigated to our knowledge probably because complex dynamic models and complex observer design make the controller design analytically and computationally prohibitive. Since a much higher order model is needed to describe the complicated dynamics of M4 compared to that of a point absorber and the wave excitation forces in more than one dimensions need to be estimated at the same time, the wave excitation force estimator design becomes more challenging. The simulations show the efficacy of the proposed ASMO in reduced estimation errors. The 1/40th scaled model of M4 during a tank testing is shown in Fig. 1, with more details introduced in Section II.

The rest of the paper is as follows. Section II introduces the state space model of M4. The ASMO for wave excitation force estimation is designed in Section III, where the ASMO is proven to be finite-time stable. An improved AR model is introduced and the boundary of the prediction error is formulated in Section IV. Section V shows the results based on realistic sea wave data gathered from Cornwall, UK, after scaling by testing both the conventional KF method and the proposed ASMO on the hydrodynamic model. Section VI concludes this paper.

\section{State Space Model of M4}

In this section, the detailed information of the device M4 is presented. The dynamical model of M4 is also given and transferred to a state space model [19] for the observer design purpose.

\section{A. Introduction to M4}

M4 is a multi-motion multi-float WEC with a complex structure. With the bow and mid-float connected by a beam to form one rigid body, the stern float connected by a beam to hinge point as another rigid body, the device enables a significant relative rotation about the hinge point when it is aligned to the wave propagating direction. The power take-off (PTO) unit above the hinge point is designed to react against the body rotation in order to absorb kinetic energy. As shown in Fig. 1, M4 is reconfigurable. Different reconfigurations can be built up to adapt different wave conditions by changing the number of mid and stern floats. The geometry of a laboratory scale (1:40) three-float M4 is demonstrated in Fig. 2. From left to right, the bow float, middle float, stern float, beam connecting bow and middle float, beam connecting middle and stern float, and the PTO unit are indexed by $i=1,2,3,4,5,6$ respectively. The 1-1-1 (numbers indicate the number of bow, mid and stern float) format M4 is adopted here for the sake of mathematical simplicity.

\section{B. Dynamical model of $M 4$}

In a laboratory experiment of M4 [9], only single direction wave (in surge direction) is considered. The device is moored properly into the wave direction, and roll motion of the system is prevented by outrigger buoys added to the stern float. Then for simplicity and consistency, this paper only focuses on the motion in $x-o-z$ plane.

Define a state vector as

$$
q=\left[\begin{array}{llll}
q_{x_{0}} & q_{z_{0}} & q_{\theta_{1}} & q_{\theta_{2}}
\end{array}\right]^{\mathrm{T}}
$$

where $q_{x_{0}}$ is the surge position of the hinge $O ; q_{z_{0}}$ is the heave position of the hinge $O ; q_{\theta_{1}}$ is the pitch angle of $i$ which is on the left of the hinge $O(i=1,2,4)$ and $q_{\theta_{2}}$ is the pitch angle of $i$ which is on the right of the hinge $O(i=3,5,6)$.

By Newton's second law, the dynamic equation for M4 is

$$
\left(m_{s}+m_{\infty}\right) \ddot{q}=f_{s}+f_{r}+f_{e}+f_{u}
$$




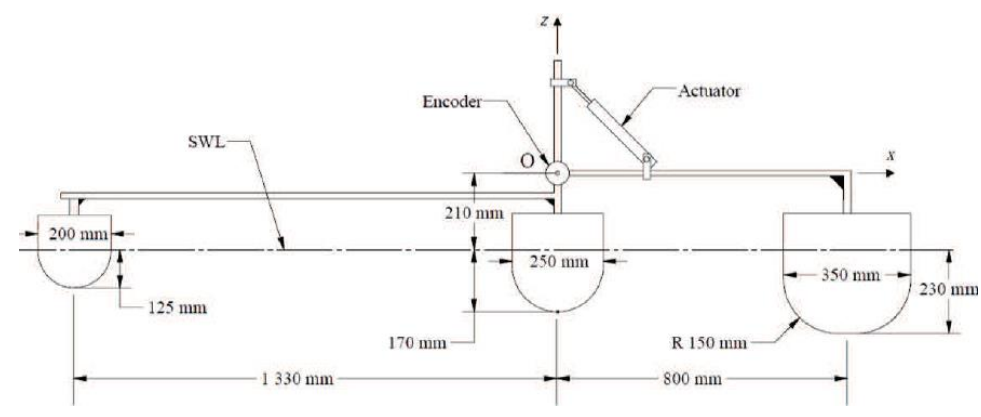

Fig. 2. Diagram of laboratory scale three-float 1-1-1 M4 [9]

$$
m_{s}=\left[\begin{array}{cccc}
\sum_{i=1}^{6} m_{i} & 0 & -m_{1} v_{1}-m_{2} v_{2}-m_{4} v_{4} & -m_{3} v_{3}-m_{5} v_{5}-m_{6} v_{6} \\
0 & \sum_{i=1}^{6} m_{i} & m_{1} h_{1}+m_{2} h_{2}+m_{4} h_{4} & -m_{3} h_{3}-m_{5} h_{5}-m_{6} h_{6} \\
-m_{1} v_{1}-m_{2} v_{2}-m_{4} v_{4} & m_{1} h_{1}+m_{2} h_{2}+m_{4} h_{4} & \sum_{i=1,2,4}\left(I_{i}+m_{i}\left(h_{i}^{2}+v_{i}^{2}\right)\right) & 0 \\
-m_{3} v_{3}-m_{5} v_{5}-m_{6} v_{6} & -m_{3} h_{3}-m_{5} h_{5}-m_{6} h_{6} & 0 & \sum_{i=3,5,6}\left(I_{i}+m_{i}\left(h_{i}^{2}+v_{i}^{2}\right)\right)
\end{array}\right]
$$

where $m_{\infty}$ is the added mass when the frequency approaches infinity, which is an invertible $4 \times 4$ matrix calculated by the hydrodynamic software WAMIT [20]; $f_{s}, f_{r}, f_{e}$ and $f_{u}$ are $4 \times 1$ vectors denoting the restoring force, the radiation force, the excitation force and the PTO force, respectively; $m_{s}$ is the mass of the device, which is an invertible $4 \times 4$ matrix expressed in (3), where $h_{i}$ and $v_{i}$ denote the horizontal and vertical distances from center of gravity (COG) of $i$ to hinge $O$ and $m_{i}$ represents the mass of the float indexed by $i$, with $i=1,2,3,4,5,6$.

The restoring force $f_{s}$ can be calculated by

$$
\begin{aligned}
f_{s} & =K q \\
K & =\left[\begin{array}{cccc}
0 & 0 & 0 & 0 \\
0 & \sum_{i=1}^{3} k_{z i} & k_{z 1} h_{1}+k_{z 2} h_{2} & -k_{z 3} h_{3} \\
0 & k_{z 1} h_{1}+k_{z 2} h_{2} & \sum_{i=1}^{2} k_{r i}+k_{z i} h_{i}^{2} & 0 \\
0 & -k_{z 3} h_{3} & 0 & k_{r 3}+k_{z 3} h_{3}^{2}
\end{array}\right]
\end{aligned}
$$

where $k_{z i}=-\rho g \pi R_{i}^{2}, k_{r i}=-\rho g \pi \frac{R_{i}^{4}}{4}$ are the restoring coefficients for heave force and pitch moment of float $i, \rho$ is the water density, $g=9.807 \mathrm{~m} / \mathrm{s}^{2}$ is the gravitational acceleration and $R_{i}$ is the radius of the float $i$ with $i=1,2,3,4,5,6$.

The radiation force $f_{r}$ can be calculated by the Cummins equation [21] as follows

$$
f_{r}=-\int_{-\infty}^{\infty} h_{r}(\tau) \dot{q}(t-\tau) d \tau-m_{\infty} \ddot{q}
$$

where $h_{r}$ is the impulse response function of the radiation force which is a $4 \times 4$ matrix. For a state-space realization, the convolution term $F_{r}=-\int_{-\infty}^{\infty} h_{r}(\tau) \dot{q}(t-\tau) d \tau$ can be considered as a linear system and be approximated by the following state-space model [4]

$$
\left\{\begin{array}{l}
\dot{r}(t)=A_{r} r+B_{r} \dot{q} \\
F_{r}(t)=C_{r} r+D_{r} \dot{q}
\end{array}\right.
$$

Here $\left(A_{r}, B_{r}, C_{r}, D_{r}\right)$ is calculated by Prony's method [22], and $r$ is a state without physical meanings whose dimension is $n_{r} \times 1$ with $n_{r}$ a positive integer.
The excitation force can be expressed as $f_{e}=$ $\left[f_{e}^{\left(x_{0}\right)}, f_{e}^{\left(z_{0}\right)}, f_{e}^{\left(\theta_{1}\right)}, f_{e}^{\left(\theta_{2}\right)}\right]^{\mathrm{T}}$, which is to be estimated in this paper.

The PTO moment $M_{\text {mech }}(t)$ is modelled linearly as $M_{\text {mech }}(t)=-B_{\text {mech }} \dot{\theta}_{r}(t)$, where $B_{\text {mech }}$ is a constant coefficient and $\dot{\theta}_{r}:=\dot{\theta}_{1}-\dot{\theta}_{2}$ is the relative pitch rotation velocity. The generalized PTO moment can be viewed as a manipulable control input to the whole system at the controller design stage.

$$
f_{u}(t)=\left[\begin{array}{c}
0 \\
0 \\
-M_{\text {mech }}(t) \\
M_{\text {mech }}(t)
\end{array}\right]
$$

\section{State-space model of M4}

Combining (4), (5) and (6), we have the following statespace model of M4

$$
\left\{\begin{array}{l}
M \ddot{q}=-K q+C_{r} r+D_{r} \dot{q}+f_{e}+f_{u} \\
\dot{r}(t)=A_{r} r+B_{r} \dot{q} \\
\dot{F}_{r}(t)=C_{r} r+D_{r} \dot{q}
\end{array}\right.
$$

where $M:=m_{s}+m_{\infty}$ is an invertible $4 \times 4$ matrix.

The system states are chosen as $x_{1}:=q, x_{2}:=r$ and $x_{3}:=\dot{q}$, and the control input is the PTO force, i.e. $u:=f_{u}$.

Substituting the system state $x=\left[\begin{array}{lll}x_{1}^{\mathrm{T}} & x_{2}^{\mathrm{T}} & x_{3}^{\mathrm{T}}\end{array}\right]^{\mathrm{T}}=$ $\left[q^{\mathrm{T}} r^{\mathrm{T}} \dot{q}^{\mathrm{T}}\right]^{\mathrm{T}}$ into (8), we have the following state-space model

$$
\dot{x}=A x+B\left(u+f_{e}\right)
$$

where

$$
\begin{gathered}
A=\left[\begin{array}{ccc}
0_{4 \times 4} & 0_{4 \times n_{r}} & I_{4} \\
0_{4 \times 4} & A_{r} & B_{r} \\
-M^{-1} K & -M^{-1} C_{r} & -M^{-1} D_{r}
\end{array}\right] \\
B=\left[\begin{array}{lll}
0_{4 \times 4} & 0_{n_{r} \times 4} & M^{-1}
\end{array}\right]^{\mathrm{T}}
\end{gathered}
$$

where $M^{-1}$ denotes the inverse matrix of $M, 0_{z_{1} \times z_{2}}$ denotes the zero matrix with the size of $z_{1} \times z_{2}$, and $I_{z_{1}}$ is an identity with the size of $z_{1} . z_{1}$ and $z_{2}$ are positive integers.

This paper aims to robustly estimate four components of the unknown wave excitation force $f_{e}$ based on the state space 


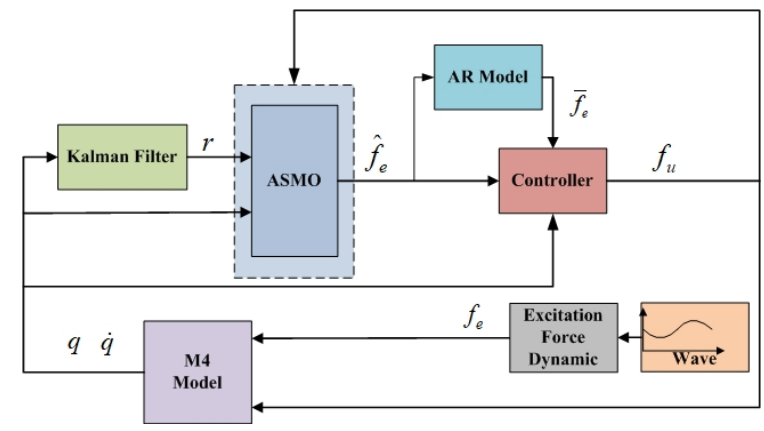

Fig. 3. Block diagram of the proposed wave excitation force estimation framework

model (9) and ensure the estimation error to be limited in a small region within the convergence time that can be explicitly specified.

\section{AdAPtive Sliding-Mode ObSERVER For WAVE EXCITATION FORCE ESTIMATION}

In this section, an adaptive sliding-mode observer (ASMO) is proposed to achieve the real-time estimation of the wave excitation force, as shown in Fig. 3. The states $q$ and its first derivative $\dot{q}$ can be directly measured, and the state corresponding to the radiation force $r$ which does not have physical meanings can be estimated by a KF [8]. By using the control input, the state $q$, its derivative $\dot{q}$ and the state $r$, the proposed ASMO estimates wave excitation force in realtime. A parameter tuning precedure of the proposed observer is also provided. For non-causal controllers, an Auto-Regressive model is adopted to predict the future excitation force, whose details are shown in Section IV.

\section{A. Wave Excitation Force Observer Design}

In this subsection, a novel SMO is designed which ensures a pre-defined convergence time and an explicit formulation of the estimation error.

In the sequel, $v^{(Q)}$ denotes the $Q$-component of the vector $v$ with $Q=x_{0}, z_{0}, \theta_{1}, \theta_{2}$.

Hypothesis 1. The wave excitation force/moment of each component and its first time derivative are bounded by known constants, so that $\left|f_{e}^{(Q)}\right| \leq F_{e}^{(Q)}$ and $\left|\dot{f}_{e}^{(Q)}\right| \leq \beta^{(Q)}$ hold with $F_{e}^{(Q)}$ and $\beta^{(Q)}$ as known positive constants.

Define a sliding variable vector as $s=\dot{q}-\zeta$. The $4 \times 1$ vector $\zeta$ is designed according to (9) as

$M \zeta=\int\left(f_{u}+\hat{f}_{e}+\mu_{0} s^{\alpha}+\mu_{1} \operatorname{sgn}(s)-K q-D_{r} \dot{q}-C_{r} r\right) d t$

where $\hat{f}_{e}=\left[\hat{f}_{e}^{\left(x_{0}\right)}, \hat{f}_{e}^{\left(z_{0}\right)}, \hat{f}_{e}^{\left(\theta_{1}\right)}, \hat{f}_{e}^{\left(\theta_{2}\right)}\right]^{\mathrm{T}}$ is a vector of the estimated wave excitation force, $\mu_{0}>0$ and $\alpha>1$ are constants and $\mu_{1}$ is designed as

$$
\mu_{1}=\left\|\hat{f}_{e}\right\|+F_{e}+k_{1}
$$

where $F_{e}=\max \left\{F_{e}^{(Q)}\right\}$ and $k_{1}>0$ is a constant.
The wave excitation force observer is proposed in the following form

$$
\left\{\begin{array}{l}
\hat{f}_{e}=\lambda+M \dot{q} \\
\lambda=\int\left(K q+D_{r} \dot{q}+C_{r} r-f_{u}-\hat{f}_{e}+\left(\hat{\beta}+K_{\mu}\right) \operatorname{sgn}(s)\right) d t
\end{array}\right.
$$

where $\lambda \in \mathbb{R}^{4}$ is an intermediate variable whose dimension is 4 and $K_{\mu}=\mu_{2} I_{4}$ is a constant matrix with $\mu_{2}>0$ a constant. $\hat{\beta}=\operatorname{diag}\left\{\hat{\beta}^{\left(x_{0}\right)}, \hat{\beta}^{\left(z_{0}\right)}, \hat{\beta}^{\left(\theta_{1}\right)}, \hat{\beta}^{\left(\theta_{2}\right)}\right\}$ is the matrix of the boundary estimation of the wave excitation force variation.

The adaptive law for the $Q$-component is designed as

$$
\dot{\hat{\beta}}^{(Q)}=-k_{2} \hat{\beta}^{(Q)}+2\left|\hat{f}_{e}^{(Q)}\right|+2 F_{e}^{(Q)}
$$

where $k_{2}>0$ is a constant and the initial value is $\beta^{(Q)}\left(t_{0}\right)=$ 0 .

The convergence process of the proposed ASMO consists of two stages: At the first stage, the estimation error of the wave excitation force is driven to approach the sliding manifold $s=0$ within a constant fixed time. At the second stage, the estimation error keeps sliding on the sliding manifold until it reaches the origin in a finite time. The convergence properties of both of the first and second stages are proven in the next two subsections respectively.

\section{B. Fixed-time stabilization of the sliding variable}

Fixed-time stabilization theory is a recently developed control theory [23], whose main advantage is that it can drive the system state to the equilibrium point within a pre-defined time which does not depend on initial states. This theory is applied in this paper to explicitly formulate the convergence time and the convergence boundary of the estimation error. The difference between finite-time convergence and fixed-time convergence is that fixed-time convergent theory ensures all the possible convergence time to be bounded by a known constant.

Lemma 1. (Lyapunov function of fixed-time stabilization) [23]. Consider the system (2). Suppose that there exists a Lyapunov function $V_{a}(x)$ defined on the neighborhood $\mathbb{U} \subset \mathbb{R}^{n}$ of the origin, and the condition

$$
\dot{V}_{a}(x) \leq-\left(\gamma_{1} V_{a}(x)^{p}+\gamma_{2} V_{a}(x)^{g}\right)^{k}
$$

is satisfied, where $\gamma_{1}, \gamma_{2}, p, k>0, p k<1$ and $g k>1$. Then the origin of the system (2) is fixed-time stable, and any $V_{a}(x)$ can reach $V_{a}(x) \equiv 0$ in a fixed time of $T_{a}$, which is bounded and independent on initial states:

$$
T_{a} \leq T_{a M A X}=\frac{1}{\gamma_{1}^{k}(1-p k)}+\frac{1}{\gamma_{2}^{k}(g k-1)}
$$

Theorem 1. For the system (9) and the sliding variable defined in (10) satisfying Hypothesis 1, the sliding variable $s$ is steered to its origin where $s=\dot{s}=0$ holds. The convergence time of the first stage $t_{1}-t_{0}$ is bounded by

$$
t_{1}-t_{0} \leq T_{1}=\frac{1}{k_{1}} \lambda_{\text {max }}^{\frac{1}{2}}(M)+\frac{1}{\mu_{0}(\alpha-1)} \lambda_{\text {max }}^{\frac{\alpha+1}{2}}(M)
$$

which is a constant with any initial state $s\left(t_{0}\right)$, where $t_{0}$ is the initial time, $t_{1}$ is the time of end of the first stage and 
$\lambda_{\max }(M)$ denotes the largest eigenvalue of the matrix $M$ which is a positive constant.

Proof: From (9) and (10), we have

$$
M \dot{s}=\tilde{f}_{e}-\mu_{0} s^{\alpha}-\mu_{1} \operatorname{sgn}(s)
$$

where $\tilde{f}_{e}=f_{e}-\hat{f}_{e}$ is the estimation error of the wave excitation force.

Select a Lyapunov candidate as

$$
V_{1}=s^{\mathrm{T}} M s
$$

Its first derivative is

$$
\begin{aligned}
\dot{V}_{1} & =2 s^{\mathrm{T}} M \dot{s} \\
& =2 s^{\mathrm{T}}\left(\tilde{f}_{e}-\mu_{0} s^{\alpha}-\mu_{1} \operatorname{sgn}(s)\right) \\
& \leq-2 \mu_{0}\|s\|^{\alpha+1}-2 \mu_{1}\|s\|+2 s^{\mathrm{T}} \tilde{f}_{e} \\
& \leq-2 \mu_{0}\|s\|^{\alpha+1}-2\left(\mu_{1}-\left\|\tilde{f}_{e}\right\|\right)\|s\| \\
& \leq-2\left(\mu_{1}-\left\|f_{e}\right\|-\left\|\hat{f}_{e}\right\|\right)\|s\|-2 \mu_{0}\|s\|^{\alpha+1}
\end{aligned}
$$

Substituting (11) into (19) and considering Hypothesis 1, we have

$$
\begin{aligned}
\dot{V}_{1} & \leq-2 k_{1}\|s\|-2 \mu_{0}\|s\|^{\alpha+1} \\
& \leq-2 k_{1} \lambda_{\max }^{\frac{1}{2}}(M) V_{1}^{\frac{1}{2}}-2 \mu_{0} \lambda_{\max }^{-\frac{\alpha+1}{2}}(M) V_{1}^{\frac{\alpha+1}{2}}
\end{aligned}
$$

Based on Lemma 1, the boundary of the convergence time can be calculated by (16).

Remark 1. Theorem 1 plays an important role in real scenarios. The initial state of the sliding variable $s\left(t_{0}\right)$ changes with respect to $\dot{q}\left(t_{0}\right)$, which leads to different convergence time. To ensure that the convergence time is available in advance, it should be bounded by a known constant with any initial states. Theorem 1 offers this upper boundary determined by (16) of all the possible convergence time with every initial state $s\left(t_{0}\right)$ and every initial state $\dot{q}\left(t_{0}\right)$. More importantly, this boundary is available once the constant parameters of the ASMO are fixed, which can be calculated in advance.

\section{Finite-time stabilization of the estimation error}

This subsection is to prove that the estimation error $\tilde{f}_{e}^{(Q)}$ converges within a region of the origin, $\tilde{f}_{e}^{(Q)}=0$, in a finite time after the sliding variable reaches $s=\dot{s}=0$.

Theorem 2. For the system (9) and the proposed observer (12) satisfying Hypothesis 1, the estimation error of the wave excitation force of the $Q$-component converges to the following region

$$
\Xi^{(Q)}=\left\{\tilde{f}_{e}^{(Q)}|| \tilde{f}_{e}^{(Q)} \mid \leq \frac{k_{2}+4 k_{2}\left(\beta^{(Q)}\right)^{2}}{8\left(1-k_{3}\right) \min \left\{2 \mu_{2}, k_{2} / \sqrt{2}\right\}}\right\}
$$

in a finite time

$$
t_{2}-t_{0} \leq T_{2}=T_{1}+\frac{2\left(V_{2}^{(Q)}\right)^{\frac{1}{2}}\left(t_{1}\right)}{k_{3} \min \left\{2 \mu_{2}, k_{2} / \sqrt{2}\right\}}
$$

where $k_{2}>0$ and $0<k_{3}<1$ are constants, $t_{2}>t_{0}+T_{1}$ denotes the time in stage 2 and $V_{2}^{(Q)}$ is defined in (24).
Proof: From (12), the derivative of the estimation error of the wave excitation force can be written as $\dot{\tilde{f}}_{e}=\dot{f}_{e}-\dot{\hat{f}}_{e}=$ $\dot{f}_{e}-K q-D_{r} \dot{q}-C_{r} r+f_{u}+\hat{f}_{e}-\left(\hat{\beta}+K_{\mu}\right) \operatorname{sgn}(s)-M \ddot{q}$.

From the state space model (9), we have $M \ddot{q}=-K q-$ $D_{r} \dot{q}-C_{r} r+f_{u}+f_{e}$. Then, it follows that

$$
\dot{\tilde{f}}_{e}=\dot{f}_{e}-\tilde{f}_{e}-\left(\hat{\beta}+K_{\mu}\right) \operatorname{sgn}(s)
$$

Design a Lyapunov candidate for the $Q$ component as

$$
V_{2}^{(Q)}=\left(\tilde{f}_{e}^{(Q)}\right)^{2}+0.5\left(\tilde{\beta}^{(Q)}\right)^{2}
$$

where $\tilde{\beta}^{(Q)}=\beta^{(Q)}-\hat{\beta}^{(Q)}$ is the estimation error of the boundary of the wave excitation force variation of the $Q$ component.

Based on (23), the time derivative is

$$
\begin{aligned}
\dot{V}_{2}^{(Q)}= & 2 \tilde{f}_{e}^{(Q)} \dot{\tilde{\tilde{f}}}_{e}^{(Q)}+\tilde{\beta}^{(Q)} \dot{\tilde{\beta}}^{(Q)} \\
= & 2 \tilde{f}_{e}^{(Q)}\left(\dot{f}_{e}^{(Q)}-\tilde{f}_{e}^{(Q)}-\left(\hat{\beta}^{(Q)}+\mu_{2}\right) \operatorname{sgn}\left(s^{(Q)}\right)\right) \\
& +\tilde{\beta}^{(Q)} \dot{\tilde{\beta}}^{(Q)} \\
= & 2 \tilde{f}_{e}^{(Q)}\left(\dot{f}_{e}^{(Q)}-\tilde{f}_{e}^{(Q)}-\left(\hat{\beta}^{(Q)}+\mu_{2}\right) \operatorname{sgn}\left(s^{(Q)}\right)\right) \\
& +\hat{\beta}^{(Q)} \dot{\hat{\beta}}^{(Q)}-\beta^{(Q)} \dot{\hat{\beta}}^{(Q)} \\
= & 2 \tilde{f}_{e}^{(Q)} \dot{f}_{e}^{(Q)}-2 \hat{\beta}^{(Q)} \tilde{f}_{e}^{(Q)} \operatorname{sgn}\left(s^{(Q)}\right)-2\left(\tilde{f}_{e}^{(Q)}\right)^{2} \\
& -2 \mu_{2} \tilde{f}_{e}^{(Q)} \operatorname{sgn}\left(s^{(Q)}\right)-\tilde{\beta}^{(Q)} \dot{\hat{\beta}}^{(Q)}
\end{aligned}
$$

From (17), the estimation error $\tilde{f}_{e}^{(Q)}$ is equivalent to the term of $\mu_{1} \operatorname{sgn}\left(s^{(Q)}\right)$ if $s=\dot{s}=0$ holds based on the equivalent output injection principle [24], [25].

From the adaptive law (13), (25) can be further written as

$$
\dot{V}_{2}^{(Q)} \leq-2 \mu_{2}\left|\tilde{f}_{e}^{(Q)}\right|+k_{2} \tilde{\beta}^{(Q)} \hat{\beta}^{(Q)}
$$

Considering the inequality $k_{2} \tilde{\beta}^{(Q)} \hat{\beta}^{(Q)}=k_{2} \tilde{\beta}^{(Q)}\left(\beta^{(Q)}-\right.$ $\left.\tilde{\beta}^{(Q)}\right) \leq \frac{k_{2}}{2}\left(\left(\beta^{(Q)}\right)^{2}-\left(\tilde{\beta}^{(Q)}\right)^{2}\right)$, we have

$$
\begin{aligned}
\dot{V}_{2}^{(Q)} \leq & -2 \mu_{2}\left|\tilde{f}_{e}^{(Q)}\right|-\frac{k_{2}}{2}\left(\left(\beta^{(Q)}\right)^{2}-\left(\tilde{\beta}^{(Q)}\right)^{2}\right) \\
\leq & -\min \left\{2 \mu_{2}, \frac{k_{2}}{\sqrt{2}}\right\}\left(V_{2}^{(Q)}\right)^{\frac{1}{2}}+\frac{k_{2}}{8}+\frac{k_{2}}{2}\left(\beta^{(Q)}\right)^{2} \\
& -\left(1-k_{3}\right) \min \left\{2 \mu_{2}, \frac{k_{2}}{\sqrt{2}}\right\}\left(V_{2}^{(Q)}\right)^{\frac{1}{2}}
\end{aligned}
$$

The Lyapunov candidate $V_{2}^{(Q)}$ keeps decreasing when the estimation errors are out of the region (21). Therefore, the estimation errors of both the wave excitation force and the boundary of its variation converge to the region of the origin in a finite time (22).

Remark 2. Note that $k_{3}$ is not a tuning coefficient of the proposed observer (12) but an auxiliary parameter to describe the convergence process, i.e. the relation between the boundary of the convergence time and the boundary of the estimation error. By decreasing $k_{3}$, the estimation error $\tilde{f}_{e}$ is confined within the region (21) which keeps shrinking as time elapses. Therefore, given a specific expected boundary of the estimation error, we can calculate the value of $k_{3}$, and the corresponding convergence time is bounded by (22), which can be precalculated. 
Remark 3. The convergence time decreases with respect to an increasing value of $\mu_{0}$; When $\lambda_{\max }(M)<1$, the convergence time is reduced with larger values of $\alpha$ and $k_{1}$; when $\lambda_{\max }(M) \geq 1$, the convergence time is shortest when $\alpha=\sqrt{2 \lambda(M)+1}$; From Eqs. (21) and (22), it can be found that with a large value of $\left\{2 \mu_{2}, \frac{k_{2}}{\sqrt{2}}\right\}$, the convergence time is reduced while the convergence region is enlarged. In contract, with a smaller convergence region, the convergence time becomes longer. Therefore, one can tune these parameters in difference scenarios according to practical demands.

\section{WAVE Excitation Force Prediction}

In this section, the future information of the wave excitation force is predicted by an improved Autoregressive (AR) model, whose coefficients are updated by online training. Compared with the conventional AR model, which has been adopted for WEC wave excitation force prediction in [26], the improved AR model enhances the prediction accuracy with different sea states. Based on the improved AR model and the proposed ASMO, the boundaries of the excitation force prediction error at each future instant can also be explicitly formulated, which can provide guaranteed control performance for the non-causal controllers.

\section{A. An improved AR model}

The 1-step-ahead-prediction AR model of the $Q$-component excitation force is given as follows:

$$
\bar{f}_{e_{k \mid k-1}}^{(Q)}=\sum_{i=1}^{p}\left(\phi_{i} \hat{f}_{e_{k-i}}^{(Q)}\right)
$$

where $\bar{f}_{e_{k \mid k-1}}^{(Q)}$ is the predicted value of $f_{e_{k}}^{(Q)}$ at instant $t_{k-1}$ and $p$ is the order of the AR prediction model, $\phi_{i}$ are the AR coefficients with $i=1,2, \ldots, p$. The $h$-step-ahead prediction is obtained by using an iterative combination of 1-step-ahead predictions with $h>1$ as a integer. The effectiveness of the AR model to predict the wave excitation force has been verified in [26], where the coefficients $\phi_{i}$ are identified by an off-line training. An improved AR model is developed whose coefficients are online trained by the latest data at each $v$ step. Therefore, the improved AR model can fully account for the change of the sea states and thus improves the prediction accuracy. Since the AR model is a first-order linear model with $p$ coefficients to be updated, the training method is to solve a least square (LS) problem, which leads to a low computational burden so that the prediction method can be efficiently implemented online.

\section{B. Boundary of the prediction error}

Based on the past estimations of the wave excitation force and the past boundaries of the estimation error, the prediction errors of the wave excitation force at each future instant can be explicitly determined.

From (21) and (22), the region of the estimation error at instant $t_{e i}=i T$ with $i=\left\lceil\frac{T_{1}}{T}\right\rceil,\left\lceil\frac{T_{1}}{T}\right\rceil+1, \ldots,\left\lceil\frac{T_{1}}{T}\right\rceil+m-1$ is

$$
\left|\tilde{f}_{e}^{(Q)}(i)\right| \leq \tilde{F}_{e}^{(Q)}(i)
$$

where

$$
\tilde{F}_{e}^{(Q)}(i)=\frac{k_{2}+4 k_{2}\left(\beta^{(Q)}\right)^{2}}{8\left(1-\frac{2 \beta^{(Q)}+2 F_{e}^{(Q)}}{\left(i T-T_{1}\right) \min \left\{2 \mu_{2}, k_{2} / \sqrt{2}\right\}}\right) \min \left\{2 \mu_{2}, k_{2} / \sqrt{2}\right\}}
$$

with $T>0$ as the sampling time, and $m>1$ as the dimension of the training data.

The 1-step-ahead prediction of wave excitation force at time $t_{e}=\left(\left\lceil\frac{T_{1}}{T}\right\rceil+m\right) T$ is calculated at time $t_{e}=\left(\left\lceil\frac{T_{1}}{T}\right\rceil+m-1\right) T$ by

$$
\bar{f}_{e}^{(Q)}\left(\left\lceil\frac{T_{1}}{T}\right\rceil+m \mid\left\lceil\frac{T_{1}}{T}\right\rceil+m-1\right)=\sum_{j=1}^{p}\left(\Phi_{j} \hat{f}_{e}^{(Q)}\left(\left\lceil\frac{T_{1}}{T}\right\rceil+m-j\right)\right)
$$

where $p$ is the order of the AR prediction model, and the coefficients $\Phi_{i}$ is trained by the estimation history of the wave excitation force $\hat{f}_{e}^{(Q)}\left(t_{e i}\right), i=\left\lceil\frac{T_{1}}{T}\right\rceil,\left\lceil\frac{T_{1}}{T}\right\rceil+1, \ldots,\left\lceil\frac{T_{1}}{T}\right\rceil+m-1$.

Note that $\hat{f}_{e}^{(Q)}\left(t_{e i}\right)$ is the estimated excitation force, based on which the prediction introduces errors. To tackle this problem, the proposed ASMO offers the boundary of the estimation error at each past estimations which enables one to calculate the boundary of the prediction error that can be compensated in the non-causal controller design.

The 1-step-ahead prediction of wave excitation force based on the actual wave excitation force can be represented by

$\bar{f}_{e}^{(Q) *}\left(\left\lceil\frac{T_{1}}{T}\right\rceil+m \mid\left\lceil\frac{T_{1}}{T}\right\rceil+m-1\right)=\sum_{j=1}^{p}\left(\Phi_{j} f_{e}^{(Q)}\left(\left\lceil\frac{T_{1}}{T}\right\rceil+m-j\right)\right)$

Combining (29), (30) and (31), we have the 1-step-ahead prediction error $\tilde{f}_{e}^{(Q)}\left(\left\lceil\frac{T_{1}}{T}\right\rceil+m \mid\left\lceil\frac{T_{1}}{T}\right\rceil+m-1\right)$ satisfying

$\left|\tilde{f}_{e}^{(Q)}\left(\left\lceil\frac{T_{1}}{T}\right\rceil+m \mid\left\lceil\frac{T_{1}}{T}\right\rceil+m-1\right)\right|=\sum_{j=1}^{p}\left(\Phi_{j} \tilde{F}_{e}^{(Q)}\left(\left\lceil\frac{T_{1}}{T}\right\rceil+m-j\right)\right)$

where

$$
\tilde{F}_{e}^{(Q)}\left(\left\lceil\frac{T_{1}}{T}\right\rceil+m-j\right)=\frac{k_{2}+4 k_{2}\left(\beta^{(Q)}\right)^{2}}{8 \min \left\{2 \mu_{2}, k_{2} / \sqrt{2}\right\}-\frac{16\left(\beta^{(Q)}+F_{e}^{(Q)}\right)}{\left(\left\lceil\frac{T_{1}}{T}\right\rceil+m-j\right) T-T_{1}}}
$$

The $h$-step-ahead prediction error can be obtained by using an iterative combination of 1-step-ahead prediction errors. The coefficients $\Phi_{j}$ are updated by training the latest $m$ dimensional estimations of the wave excitation force.

\section{Simulation Results on A Realistic Sea Wave of CORNWALL, UK}

The wave forces and the added mass of the M4 WEC are calculated based on a scaled realistic sea wave heave trajectory gathered from the coast of Cornwall, UK by WAMIT [20]. The sea wave heave trajectory is shown in Fig. 4. The corresponding wave excitation forces/moments are shown in Fig. 5 which are used to be the reference to validate the accuracy of the estimations.

In subsection $\mathrm{V}-\mathrm{A}$, for comparison purpose, four components of the wave excitation force are estimated by both the conventional KF and the proposed ASMO. First, we consider the model mismatch between the state-space model that is 


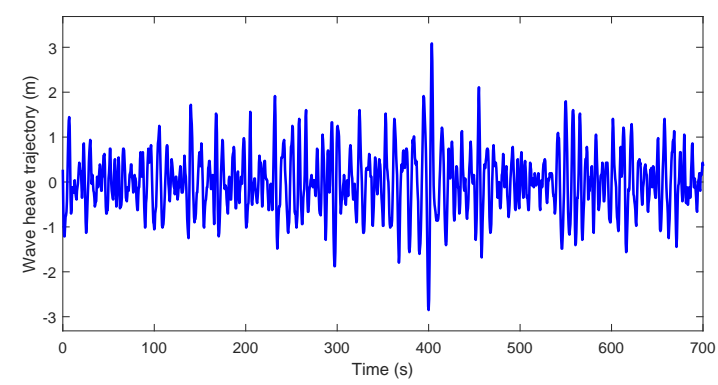

Fig. 4. A realistic sea wave heave trajectory gathered from the coast of Cornwall, UK
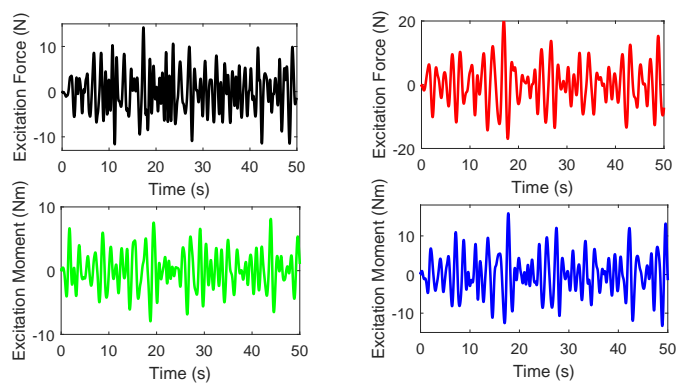

Fig. 5. Real excitation force/moment of the wave shown in Fig. 4

used by the proposed ASMO and the original hydrodynamic model that is the M4 plant. Therefore, the robustness of the estimators is tested with this model mismatch caused by model order reduction and linearization. Second, further considering that the existence of unknown disturbances, such as unmodeled high-order wave forces and environmental uncertainties, etc., we test a case where the model mismatch is set to be $30 \%$ in order to fully demonstrate the robustness of the proposed ASMO.

In subsection V-B, the wave excitation force prediction based on the improved AR model is validated. The historical data is composed of the estimations of the estimators, based on which the predictions of the excitation force are generated by AR model. By using the proposed ASMO, the prediction error at each future step is verified to be within the pre-calculated boundary, which provides guaranteed control performance for the design of non-causal controllers.

\section{A. Validation of Estimation Accuracy}

1) Model mismatch caused by model order reduction and linearization: The estimations using both the conventional KF and the proposed ASMO are shown in Fig. 6.

The simulation results are summarized in Table I. The performance indexes are the maximum error (ME) of the wave excitation force estimation calculated by

$$
\mathrm{ME}=\max \left\{\left|f_{e}(i)-\hat{f}_{e}(i)\right|\right\}, i=1, \ldots, N
$$

the average error (AE) of estimation calculated by

$$
\mathrm{AE}=1 / N \sum_{i=1}^{N}\left|f_{e}(i)-\hat{f}_{e}(i)\right|
$$
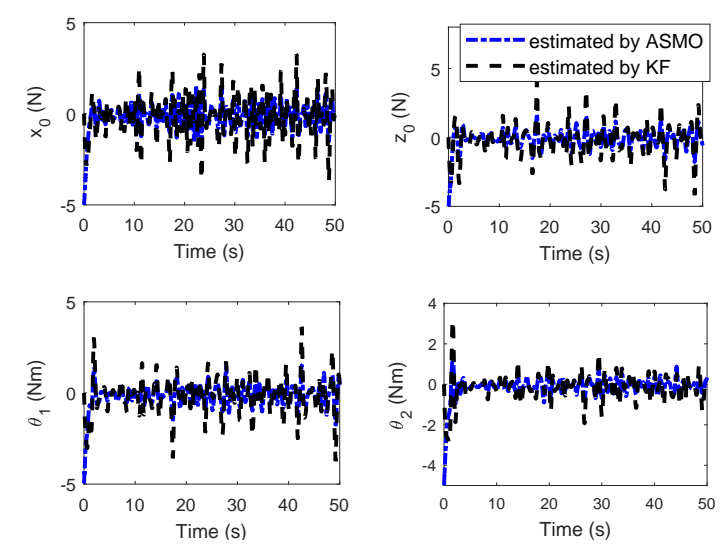

Fig. 6. Estimation errors of wave excitation forces

TABLE I

SiMULATION RESULT COMPARISON

\begin{tabular}{|l|l|l|l|l|l|}
\hline & $\begin{array}{l}\text { Performance } \\
\text { Index }\end{array}$ & $x_{0}$ & $z_{0}$ & $\theta_{1}$ & $\theta_{2}$ \\
\hline KF & ME & 4.02 & 4.38 & 3.08 & 4.11 \\
& AE & 2.08 & 1.96 & 1.22 & 1.92 \\
& NMSE & $84.28 \%$ & $92.24 \%$ & $85.85 \%$ & $90.51 \%$ \\
\hline ASMO & ME & 5.00 & 5.00 & 5.00 & 5.00 \\
& AE & 1.32 & 1.26 & 0.87 & 1.19 \\
& NMSE & $97.5 \%$ & $98.82 \%$ & $96.94 \%$ & $98.46 \%$ \\
\hline
\end{tabular}

and the normalized mean square error (NMSE) calculated by

$$
\operatorname{NMSE}=\left(1-\sum_{i=1}^{N}\left(f_{e}(i)-\hat{f}_{e}(i)\right)^{2} / \sum_{i=1}^{N} f_{e}^{2}(i)\right) 100 \%
$$

Here $N$ is the number of data. Compared with the conventional $\mathrm{KF}$, the proposed ASMO has a smaller steady-state estimation error. Although the estimation error of the ASMO is larger than that of the conventional KF at the very beginning, a warming-up process can be operated in the real scenario to initialize the device, so that the proposed ASMO can converge to the steady state and estimates the wave excitation force with small errors thereafter. Therefore, considering that the steadystate performance of an observer is the most important index to evaluate its effectiveness and superiority in application to WECs, we conclude that the proposed ASMO outperforms the conventional KF.

2) 30\% Modelling mismatch: We consider a harsh situation where the model mismatch of $30 \%$ from the original hydrodynamic model exists in order to investigate the robustness of the proposed ASMO. The estimation errors of the conventional KF and the ASMO are shown in Fig. 7. Simulation results are concluded in Table II. Compared with the conventional KF, the proposed ASMO has a smaller estimation error and faster convergence speed when a large model mismatch presents. By comparing with Table I, we can find that the model mismatch has a bigger impact on the KF than the proposed ASMO. Therefore, the robustness of the proposed ASMO is verified.

\section{B. Validation of the wave excitation force prediction}

In this section, the conventional AR model and the improved AR model are used respectively to predict the wave excitation 

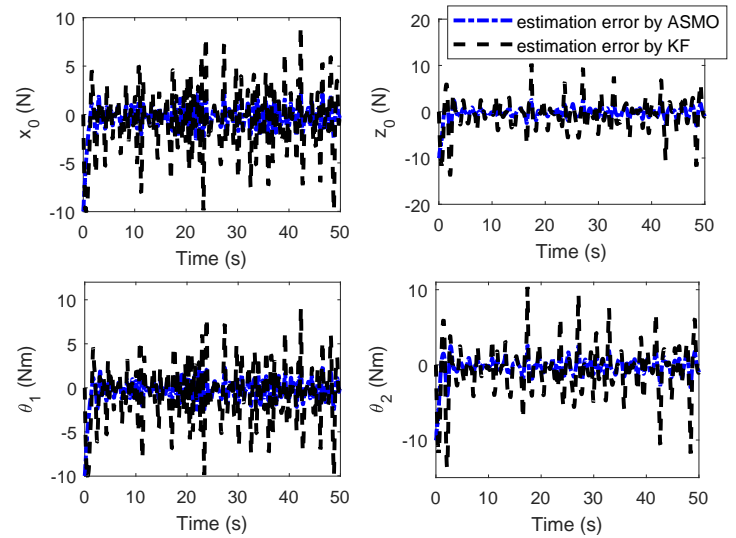

Fig. 7. Estimation errors of wave excitation force with $30 \%$ modelling mismatch

TABLE II

SiMULATION RESULT COMPARISON (30\% MODEL MISMATCH)

\begin{tabular}{|l|l|l|l|l|l|}
\hline & $\begin{array}{l}\text { Performance } \\
\text { Index }\end{array}$ & $x_{0}$ & $z_{0}$ & $\theta_{1}$ & $\theta_{2}$ \\
\hline KF & ME & 9.79 & 12.66 & 9.83 & 13.51 \\
& AE & 5.43 & 4.82 & 5.66 & 5.71 \\
& NMSE & $74.17 \%$ & $72.41 \%$ & $69.85 \%$ & $70.51 \%$ \\
\hline ASMO & ME & 10.00 & 10.00 & 10.00 & 10.00 \\
& AE & 2.05 & 3.01 & 2.97 & 2.19 \\
& NMSE & $92.33 \%$ & $94.28 \%$ & $89.08 \%$ & $94.16 \%$ \\
\hline
\end{tabular}

force, and sea waves consist of two sea states, as shown in Fig. 8. The sea state changes from sea wave (A) to sea wave (B) at $t=351.360 \mathrm{~s}$.

The orders of the conventional and improved AR models are both 25 according to the plot of the autocorrelation function $(\mathrm{ACF})$ and partial autocorrelation function $(\mathrm{PACF})$, and the dimensions of the training data of two AR models are both 200. The coefficients of the conventional AR model are obtained by offline training using the data during sea wave (A). 535 -step-ahead predictions made at $t=360.36 \mathrm{~s}$ by the conventional and the improved AR models are shown in Fig. 8. It can be found that the prediction errors of the conventional
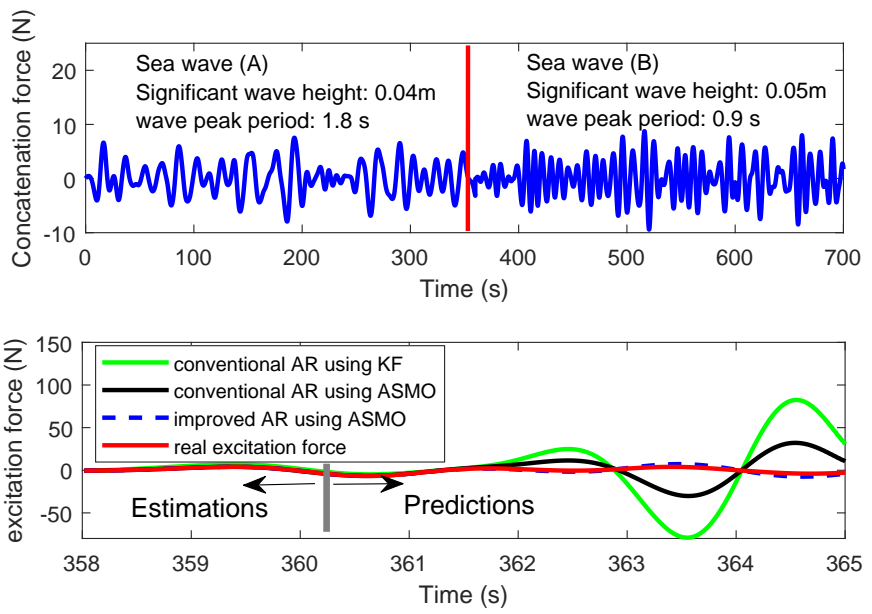

Fig. 8. 535-step-ahead AR predictions of Concatenation excitation force

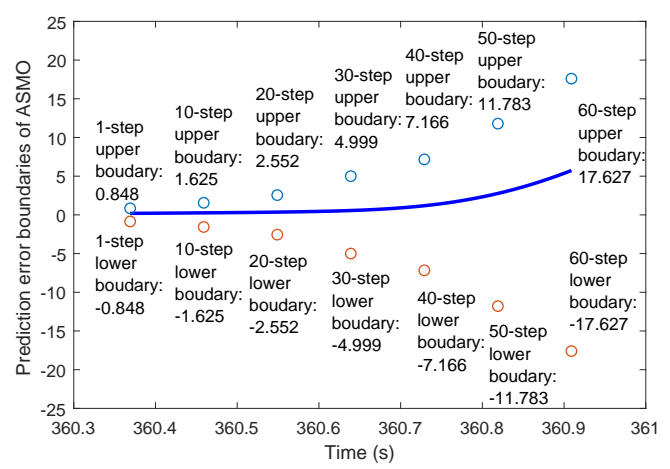

Fig. 9. 60-step-ahead prediction error boundaries (blue circles: pre-calculated upper boundaries of the prediction error; red circles: pre-calculated lower boundaries of the prediction error; the blue line: the estimation error by the proposed ASMO)

AR model are larger than those of the improved AR model. Because the improved AR model can online account for the change of the sea state. In the subsection V-A, the estimation accuracy of ASMO has been validated by comparing it with the conventional KF. Therefore, a more accurate prediction by using the historical data estimated by the ASMO can be expected when it is combined with the online adaptive AR model, as shown in Fig. 8.

From Fig. 9, it can be found that the actual prediction errors at each step are within the pre-calculated boundaries. Since the upper and lower boundaries of the prediction errors resulted from the proposed ASMO can be explicitly formulated and pre-calculated, it allows the non-causal controller design to achieve guaranteed control performance by explicitly taking into account the wave force estimation error and AR prediction error.

\section{CONCLUSIONS}

An adaptive sliding-mode observer has been proposed to estimate the wave excitation force for the wave energy converter M4. A sliding variable was designed to steer the estimation error into a pre-defined region within a pre-defined convergence time that can be defined a priori. Based on the proposed ASMO, the AR prediction error of excitation force was proven to be explicitly bounded. The results based on a realistic sea wave heave trajectory gathered from the coast of Cornwall, UK showed the effectiveness of the proposed ASMO. Future work will focus on the prediction method using deep learning.

\section{ACKNOWLEDGMENT}

This work was supported in part by a research contract from Wave Energy Scotland Control Systems programme, in part by EPSRC grant 'Launch and Recovery in Enhanced Sea States' (no. EP/P023002/1) and in part by Newton Advanced Fellowship (NA160436).

\section{REFERENCES}

[1] Zanxiang Nie, Xi Xiao, Richard McMahon, Peter Clifton, Yunxiang Wu, and Shiyi Shao. Emulation and control methods for direct drive linear wave energy converters. IEEE Transactions on Industrial informatics, 9(2):790-798, 2013. 
[2] Hany M Hasanien. Transient stability augmentation of a wave energy conversion system using a water cycle algorithm-based multi-objective optimal control strategy. IEEE Transactions on Industrial Informatics, 2018.

[3] John V Ringwood, Giorgio Bacelli, and Francesco Fusco. Energymaximizing control of wave-energy converters: The development of control system technology to optimize their operation. IEEE Control Systems Magazine, 34(5):30-55, 2014.

[4] Siyuan Zhan and Guang Li. Linear optimal noncausal control of wave energy converters. IEEE Transactions on Control Systems Technology, 2018.

[5] Francesco Fusco and John V Ringwood. Short-term wave forecasting for real-time control of wave energy converters. IEEE Transactions on sustainable energy, 1(2):99-106, 2010.

[6] Ryan Geoffrey Coe, Giorgio Bacelli, David G Wilson, Ossama Abdelkhalik, Shangyan Zou, and Rush D Robinett. Estimation of excitation force on wave energy converters using pressure measurements for feedback control. Technical report, Sandia National Lab.(SNL-NM), Albuquerque, NM (United States), 2016.

[7] Peter Kracht, Sebastian Perez-Becker, Jean-Baptiste Richard, and Boris Fischer. Performance improvement of a point absorber wave energy converter by application of an observer-based control: Results from wave tank testing. IEEE Transactions on Industry Applications, 51(4):3426$3434,2015$.

[8] M Garcia-Abril, F Paparella, and John V Ringwood. Excitation force estimation and forecasting for wave energy applications. IFACPapersOnLine, 50(1):14692-14697, 2017.

[9] Peter Stansby, Efrain Carpintero Moreno, and Tim Stallard. Large capacity multi-float configurations for the wave energy converter $\mathrm{m} 4$ using a time-domain linear diffraction model. Applied Ocean Research, 68:53 - 64, 2017.

[10] Shihua Li, Mingming Zhou, and Xinghuo Yu. Design and implementation of terminal sliding mode control method for pmsm speed regulation system. IEEE Transactions on industrial informatics, 9(4):1879-1891, 2012.

[11] Jun Yang, Jinya Su, Shihua Li, and Xinghuo Yu. High-order mismatched disturbance compensation for motion control systems via a continuous dynamic sliding-mode approach. IEEE Transactions on Industrial Informatics, 10(1):604-614, 2013.

[12] Samet Biricik and Hasan Komurcugil. Optimized sliding mode control to maximize existence region for single-phase dynamic voltage restorers. IEEE Transactions on Industrial Informatics, 12(4):1486-1497, 2016.

[13] Sanjay P Bhat and Dennis S Bernstein. Continuous finite-time stabilization of the translational and rotational double integrators. IEEE Transactions on automatic control, 43(5):678-682, 1998.

[14] Charles E Hall and Yuri B Shtessel. Sliding mode disturbance observerbased control for a reusable launch vehicle. Journal of guidance, control, and dynamics, 29(6):1315-1328, 2006.

[15] Jun-Jie Ren, Yan-Cheng Liu, Ning Wang, and Si-Yuan Liu. Sensorless control of ship propulsion interior permanent magnet synchronous motor based on a new sliding mode observer. ISA transactions, 54:15-26, 2015.

[16] Hongryel Kim, Jubum Son, Jangmyung Lee, et al. A high-speed slidingmode observer for the sensorless speed control of a pmsm. IEEE Transactions on Industrial Electronics, 58(9):4069-4077, 2011.

[17] Peter Stansby, Efrain Carpintero Moreno, Tim Stallard, A Maggi, and $\mathrm{R}$ Eatock Taylor. Wave energy conversion with high capture width by the three-float line absorber m4. Proceedings Renewable Energies Offshore, Lisbon, 2014.

[18] P Stansby, E Carpintero Moreno, T Stallard, and A Maggi. Threefloat broad-band resonant line absorber with surge for wave energy conversion. Renewable energy, 78:132-140, 2015.

[19] Zhijing Liao, Nian Gai, Peter Stansby, and Guang Li. Control-oriented modelling of wave energy converter $\mathrm{m} 4$. In The 4th Asian Wave and Tidal Energy Conference, pages 1-8, 2018.

[20] Chang-Ho Lee. WAMIT theory manual. Massachusetts Institute of Technology, Department of Ocean Engineering, 1995.

[21] WE Cummins. The impulse response function and ship motions. Technical report, David Taylor Model Basin Washington DC, 1962.

[22] Griet De Backer. Hydrodynamic design optimization of wave energy converters consisting of heaving point absorbers. Department of Civil Engineering, Ghent University: Ghent, Belgium, 2009.

[23] Andrey Polyakov. Nonlinear feedback design for fixed-time stabilization of linear control systems. IEEE Transactions on Automatic Control, 57(8):2106, 2012.

[24] Ibrahim Haskara. On sliding mode observers via equivalent control approach. International Journal of control, 71(6):1051-1067, 1998.
[25] Thierry Floquet and Jean-Pierre Barbot. Super twisting algorithm-based step-by-step sliding mode observers for nonlinear systems with unknown inputs. International journal of systems science, 38(10):803-815, 2007.

[26] Yerai Pena-Sanchez, Marina Garcia-Abril, Francesco Paparella, and John V Ringwood. Estimation and forecasting of excitation force for arrays of wave energy devices. IEEE Transactions on Sustainable Energy, 2018.

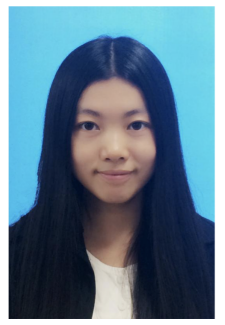

Yao Zhang received her Ph. D. in Department of Control Science and Engineering, Harbin Institute of Technology in 2018. She is currently a postdoctoral researcher in Queen Mary University of London. Her research interest covers sliding mode control, model predictive control and control applications.

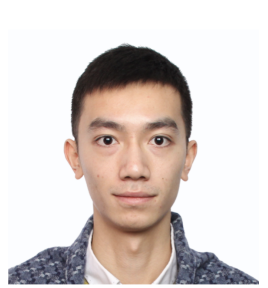

Tianyi Zeng Tianyi Zeng is with the School of Automation, Beijing Institute of Technology. He has received the M.S degree in Department of Control Science and Engineering from Harbin Institute of Technology, Heilongjiang, China, in 2015. His research interest covers motor drive control and codesign of mechanism parameters and the controller.

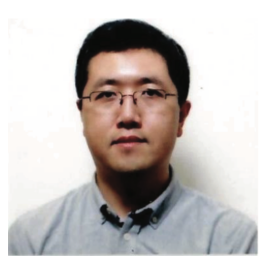

Guang $\mathbf{L i}$ received his Ph.D. degree in Electrical and Electronics Engineering, specialized in control systems, from the University of Manchester, in 2007. $\mathrm{He}$ is currently a senior lecturer in dynamics modelling and control in Queen Mary University of London, UK. His current research interests include constrained optimal control, model predictive control, adaptive robust control and control applications including renewable energies and energy storage, etc. 